\title{
Nonlinear Time Series Predication of Slope Displacement based on Smoothing Filtered Data
}

\author{
Jiawen Zhou*, Xingguo Yang, Wei Hu \\ State Key Laboratory of Hydraulics and Mountain River Engineering; Sichuan University, Chengdu, 610065, China \\ E-mail: jwzhou@ scu.edu.cn
}

\begin{abstract}
Accor ding to the slope in geotechnical engineering, many displacement monitoring points are usually set to obtain the displacement data to ensure slope stability, these data are typical nonlinear time series, and it has high value about how to make use of displacement monitoring data to do the next step for ecast analysis. Due to a certain degree of error, smoothing filter method is used to pretreat the displacement data, eliminate the influence of the error on the results and ensure the rationality. Based on smoothing filter data, these two methods are proposed to predict the displacement of the slope: exponential smoothing and chaos neural network. Both methods are used to make predictive analysis of the displacement monitoring data of outer monitoring point TP/BM 27 in high slope of Three Gorges Ship-L ock, forecasting results show that: predictive values are close to measured values, chaos neural network prediction method is better than exponential smoothing method. At the same time, the displacement data with higher reliability and smoothing filter processing are used to make predictive analysis, the results can be more reasonable, so smoothing filter processing plays an important role in the analysis of displacement prediction.
\end{abstract}

Index Terms-geotechnical engineering, monitoring data, nonlinear time series, credibility, smoothing filter, predication

\section{INTRODUCTION}

Nonlinear time series models have received a growing interest both from the theoretical and the applied points of view. Nonlinearity testing has been an active subject of research [1]. Nonlinear time series forecasting is an important area of forecasting in which past observations of the same variable are collected and analyzed to develop a model describing the underlying relationship [2]. The model is then used to extrapolate the nonlinear time series into the future. This modeling approach is particularly useful when little knowledge is available on the underlying data generating process or when there is no satisfactory explanatory model that relates the predication variable to other explanatory variables. The mathematical theory of time-delay embedding by Takens [3] and later by Sauer et al. has been provided a technique to view the nonlinear time series. Several related algorithms, such as calculation of correlation dimension

*Corresponding author. Tel.: +86-28-8540-5011; fax: +86-288540-5011.

E-mail address: jwzhou@scu.edu.cn (J.W. Zhou)

Manuscript received August 11, 2009; revised September 10, 2009; accepted September 25, 2009. and calculation Lyapunov exponents have thereafter been developed [4], which make characterizing dynamical behavior from nonlinear time series data possible.

Monitoring points are often arranged for the sake of safety during construction and running in geotechnical engineering. Generally speaking, the monitoring data behave the characteristic of nonlinear time series. For the nonlinear time series, pre-filtering is essential for removing time trends or noises. The most commonly used filters are linear filters, e.g. the HP filter [5], the BandPass filter [6], and the moving average filters. The popularity of linear filters is due mainly to the sound theoretical basis provided by the theory of linear systems. For the slope displacement data, linear filter can removing the noises of nonlinear time series effectively.

To predict the future trend by using these monitoring data series is significant [7]. However, the monitoring data in geotechnical engineering is a complicated multifactor influenced nonlinear dynamic system; it must be very difficult to establish nonlinear dynamic equation to analyses monitoring data $[8,9]$. Nonlinear time series prediction methods are developed since 1980s of the 20th century. These methods are widely applied to predict short-term electric load, share price, weather and the displacement of slope [10 14].

To predict the slope displacement monitoring data, first of all, the pretreatment of the displacement data should be analyzed, including the credibility analysis of data and smoothing filter processing, and then based on the smoothing filtered data, two types of displacement prediction methods are proposed: exponential smoothing and chaos neural network.

These two methods are applied to predict the displacement of outer monitoring point TP/BM27 in the 17-17 section in high slope of Three Gorges Permanent Ship-Lock. Finally, the forecast results are carried on a comparative analysis under different conditions, and some meaningful conclusions are obtained.

\section{DisPlacement DATA PREPROCESSING}

Affected by the observing conditions, errors may exist in any displacement monitoring data, only the size and the type of error are different. In the monitoring process, the errors of the monitoring value are divided into three categories: (1) Mistakes, which is due to observational errors, such as read or write errors. (2) Systematical errors, a series of observations are made in the same conditions, and observation errors show systematic 
characteristic in size and symbol, such as fixed error of the distance gauge; (3) Occasional errors, also known as random errors, a series of observations are made in the same conditions, and observation errors show contingency characteristic in size and symbol, such as decimal estimate errors. In the course of displacement monitoring, observation mistakes are not allowed, systematical errors can be eliminate or weaken through certain procedures, but it is difficult to avoid occasional errors. For these reasons, first of all, credibility analysis of the displacement monitoring data of the slope should be carried out to determine the reliability of data, and then linear smoothing filter method should be applied to handle and revise the data.

\section{A. Reliability Analysis}

The credibility is computed by fuzzy sets [15], its main idea is: $U$ is domain, fuzzy sets $A$ is expressed by a realvalued function in $U: u_{A}: U \rightarrow[0,1] . u \in U$ and $u_{A}(u)$ are defined a degree of membership of $u$ to $A$, and $u_{A}$ is defined a membership function of $A$.

Based on the above-mentioned concept of fuzzy sets, the correctness of the displacement monitoring data can be seen as a fuzzy set $A$, the domain $U$ is the displacement monitoring data of the slope, thus there is a affiliation relationship of $u_{A}(u)$, that is, a degree of membership of $U$ to $A$ and the credibility of the displacement monitoring data. The credibility indicates the reliability of monitoring data, using $k$ to express, the definition interval is $[0,1], 0$ expressed that the credibility is the minimum, that is, the degree of the data affiliated to $A$ is the lowest, 1 expressed that the credibility is the maximum, that is, the degree of the data affiliated to $A$ is the highest.

The sequence of the displacement data is hypothesized as: $X_{1}, X_{2}, L, X_{i}, L x_{n}$, so the data lies in the location of $\mathrm{i}$ is $\mathrm{x}_{\mathrm{i}}$,previous data is $\mathrm{x}_{\mathrm{i}-1}$, differentials $\Delta \mathrm{x}_{\mathrm{i}}$ between the two data is,

$$
\Delta \mathrm{x}_{\mathrm{i}}=\mathrm{x}_{\mathrm{i}}-\mathrm{x}_{\mathrm{i}-1}
$$

The number of displacement data is $n$, so the amount of the monitoring data differentials is $n-1$, the average of all monitoring data differentials is calculated,

$$
\overline{\Delta x}=\frac{1}{n-1} \sum_{i=1}^{n-1} \Delta x_{i}
$$

$R_{i}$ that defined as the ratio of each margin $\Delta x_{i}$ and the average of differentials $\overline{\Delta x}$ is calculated,

$$
\mathrm{R}_{\mathrm{i}}=\frac{\Delta \mathrm{x}_{\mathrm{i}}}{\overline{\Delta \mathrm{x}}} \quad(\mathrm{i}=1,2, \mathrm{~L}, \mathrm{n}-1)
$$

A nonlinear function about $R_{i}$ is defined: $f\left(R_{i}\right)$, $f\left(R_{i}\right) \in[0,1]$, that is $k$ which is the credibility of the displacement monitoring data, the credibility of each point is calculated, then can be obtained all. At the same time, cut-off point of the credibility can be defined,

$$
\left\{\begin{array}{l}
\text { uncredibility, if } 0 \leq k \leq 0.5 \\
\text { credibility, if } 0.5<k \leq 1
\end{array}\right.
$$

There is, if the credibility is more than 0.5 , the data is reliable; if the credibility is less than 0.5 , the data is unreliable.

Take a simple example to explain, Fig. 1 is a displacement monitoring time series, Fig.2 is the credibility of the upper data.

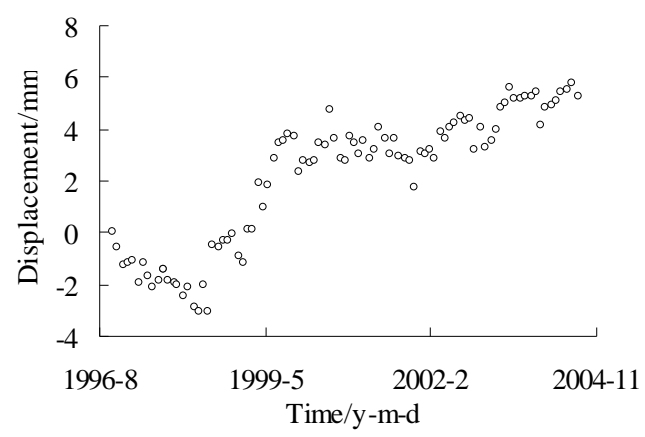

Figure 1. Time series of monitor displacement

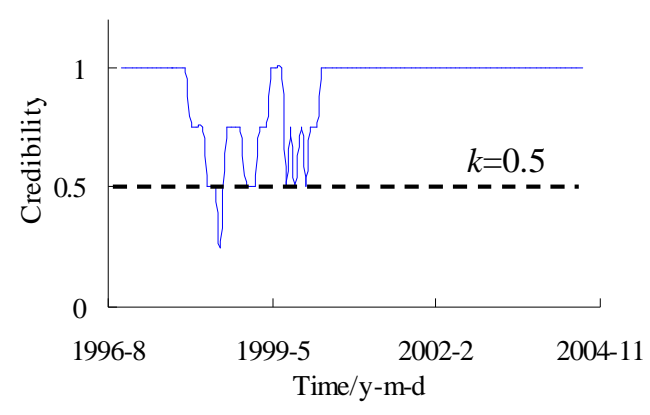

Figure 2. Compute result of credibility

As can be seen from Fig.2, the majority of the credibility of the displacement data are more than 0.5 , only a small number are less than 0.5 , so whole of the monitoring data are more reliable.

\section{B. Data smoothing}

The displacement data obtained during the monitoring process is often superposed by the true signal that we need and a variety of interference, error or noise. In order to preserve the true signal and eliminate the noise from the actual monitoring data, the linear smoothing filter method [16] is used to deal with the monitoring data.

Observation object is $\mathrm{x}$, the corresponding variable is $t, \Delta t$ is hypothesized as the distance of $t$, based on these numerical, observing the values of the dependent variable $x$, observational data sequence is obtained: $\mathrm{X}_{1}, \mathrm{X}_{2}, \mathrm{~L}, \mathrm{X}_{\mathrm{i}}, \mathrm{L} \mathrm{x}_{\mathrm{n}}$. Smoothing filter can be use to filter the noise components of $x_{i}(i=1,2, L, i, L, n)$. The principle is that: Take the point of $i$ and a number of data in the vicinity of it, a fitting line equation is determined according to the principle of least-squares, and then it can calculate the smoothing sliding value of the dependent variable i . Generally speaking, there are three-point linear smoothing filter, five-point linear smoothing filter, seven-point linear smoothing filter and m-point linear smoothing filter, here five-point linear smoothing filter is used. 
For any point of $i$, a total of five data points which comprised by itself and other data before and after it are choice: $\left(t_{i-2}, x_{i-2}\right),\left(t_{i-1}, x_{i-1}\right),\left(t_{i}, x_{i}\right),\left(t_{i+1}, x_{i+1}\right)$, $\left(t_{i+2}, x_{i+2}\right)$. Firstly these five data points are fitted with linear equation in accordance with the principle of leastsquares, the basic formula of the weighted average algorithm is as follows,

$$
u_{i}=\sum_{m=-2}^{2} h_{m} x_{i-m} \quad(i=1,2, L, n)
$$

Where, $x$ is monitoring data; $u$ is the result of smoothing; $\mathrm{n}$ is amount of the data; $\mathrm{h}$ is the weighted average factor.

The weighted average factor must meet the following formula,

$$
\sum_{m=-2}^{2} h_{m}=1
$$

For the simple average method, taking

$$
h_{m}=1 / 5 \quad(m=i-2, i-1, i, i+1, i+2)
$$

Then

$$
u_{i}=\frac{1}{5} \sum_{m=-2}^{2} x_{i-m}
$$

For the weighted average method, weighted average of the five-point, taking

$$
\{h\}=\left(h_{-2}, h_{-1}, h_{0}, h_{1}, h_{2}\right)=\frac{1}{9}(1,2,3,2,1)
$$

The least-square method is used to calculate the fivepoint sliding average value of the monitoring data,

$$
\left\{\begin{array}{l}
u_{1}=\frac{1}{5}\left(3 x_{1}+2 x_{2}+x_{3}-x_{4}\right) \\
u_{2}=\frac{1}{10}\left(4 x_{1}+3 x_{2}+2 x_{3}+x_{4}\right) \\
u_{i}=\frac{1}{5}\left(x_{i-2}+x_{i-1}+x_{i}+x_{i+1}+x_{i+2}\right) \\
L \\
u_{n}=\frac{1}{5}\left(-x_{n-3}+x_{n-2}+2 x_{n-1}+3 x_{n}\right)
\end{array}\right.
$$

Following is the data smoothing that used type (6).

\section{EXPONENTIAL SMOOTHING FORECASTING MODEL}

Exponential smoothing, also known as index smoothing, is a special weighted moving average forecasting method developed from moving average forecasting method and is an important time-series prediction [17]. Exponential smoothing method is weighted combined by using the past historical data to directly predict the future value of the time series. Exponential smoothing method also can make the smoothing of the time series, it is not for the arithmetic average, but focusing common effects of the long-term value of the time series on the future predictive value ,the nearer the time draws, the greater its weight, the weighted coefficient attenuates according to geometric progression, the nearer the data draws, the greater its weight, and equal to 1 , the weighted coefficient conforms to the exponential law, and also has the function of exponential smoothing, so it is called exponential smoothing. Depending on the number of smoothing, it is divided into: single exponential smoothing, quadratic exponential smoothing, three exponential smoothing method and so on.

Based on the smoothing filter of the monitoring data, the principle of exponential smoothing is used to predict the displacement monitoring data of the slope.

\section{A. The basic principles of exponential smoothing}

For the displacement time series: $\mathrm{X}_{1}, \mathrm{x}_{2}, \mathrm{~L} \mathrm{X}_{\mathrm{t}}$, according to the moving-average method, the following formula is obtained,

$$
\begin{aligned}
S_{t} & =\frac{1}{n}\left(x_{t}+x_{t-1}+x_{t-2}+L+x_{t-n+1}\right) \\
& =\frac{1}{n} x_{t}-\frac{1}{n} x_{t-n}+S_{t-1}
\end{aligned}
$$

If the error is ignored, ordering $x_{t-n} \approx S_{t-1}$, then the formula can be written as,

$$
S_{t}=\frac{1}{n} x_{t}+\left(1-\frac{1}{n}\right) S_{t-1}
$$

Where, $n$ is equal to $1,1 / n=1$; When $n \rightarrow \infty$, $\lim _{n \rightarrow \infty} \frac{1}{n}=0 ;$ Ordering $\alpha=1 / n$, and $\alpha$ is between 0 and 1 , it is called as the smoothing factor, then the type (12) can be written as a exponential smoothing sequence recursive formula,

$$
\mathrm{S}_{\mathrm{t}}^{(1)}=\alpha \mathrm{x}_{\mathrm{t}}+(1-\alpha) \mathrm{S}_{\mathrm{t}-1}^{(1)}
$$

Where, $S_{t}^{(1)}$ is the single exponential smoothing value for the time of $t ; S_{t-1}^{(1)}$ is the single exponential smoothing value for the time of $t-1 ; x_{t}$ is actual value of the displacement monitoring.

\section{B. Quadratic exponential smoothing predication method}

Based on the first exponential smoothing, and then calculated the second exponential smoothing. Formula of the smoothing values are respectively calculated as,

$$
\left\{\begin{array}{l}
\mathrm{S}_{\mathrm{t}}^{(1)}=\alpha \mathrm{x}_{\mathrm{t}}+(1-\alpha) \mathrm{S}_{\mathrm{t}-1}^{(1)} \\
\mathrm{S}_{\mathrm{t}}^{(2)}=\alpha \mathrm{S}_{\mathrm{t}}^{(1)}+(1-\alpha) \mathrm{S}_{\mathrm{t}-1}^{(2)}
\end{array}\right.
$$

Where, $\mathrm{S}_{\mathrm{t}}^{(2)}$ is the quadratic exponential smoothing value for the time of $t ; S_{t-1}^{(2)}$ is the quadratic exponential smoothing value for the time of $t-1$.

The displacement monitoring data is predicted by using the smoothing value to establish the forecasting model, forecasting model is,

$$
Y_{t+T}^{(2)}=a_{t}+b_{t} T
$$

Where, $Y_{t+T}^{(2)}$ is the quadratic exponential smoothing predicting value of the forecast cycle $T$ for the time of $t$; $a_{t}$ and $b_{t}$ are smoothing coefficient, the formulas are, 


$$
\left\{\begin{array}{l}
\mathrm{a}_{\mathrm{t}}=2 \mathrm{~S}_{\mathrm{t}}^{(1)}-\mathrm{S}_{\mathrm{t}}^{(2)} \\
\mathrm{b}_{\mathrm{t}}=\frac{\alpha}{1-\alpha}\left(\mathrm{S}_{\mathrm{t}}^{(1)}-\mathrm{S}_{\mathrm{t}}^{(2)}\right)
\end{array}\right.
$$

The determination of value $\alpha$ can be calculated by selecting several different values of $\alpha$ according to experience, and then the smallest error value could be selected. For the determination of the initial value, the following methods are used to select,

$$
\mathrm{S}_{0}^{(2)}=\mathrm{S}_{0}^{(1)}=\mathrm{x}_{1}
$$

\section{Three exponential smoothing predication method}

Based on the quadratic smoothing, conducting another smoothing. Formula of the smoothing values is respectively calculated as,

$$
\left\{\begin{array}{l}
\mathrm{S}_{\mathrm{t}}^{(1)}=\alpha \mathrm{x}_{\mathrm{t}}+(1-\alpha) \mathrm{S}_{\mathrm{t}-1}^{(1)} \\
\mathrm{S}_{\mathrm{t}}^{(2)}=\alpha \mathrm{S}_{\mathrm{t}}^{(1)}+(1-\alpha) \mathrm{S}_{\mathrm{t}-1}^{(2)} \\
\mathrm{S}_{\mathrm{t}}^{(3)}=\alpha \mathrm{S}_{\mathrm{t}}^{(2)}+(1-\alpha) \mathrm{S}_{\mathrm{t}-1}^{(3)}
\end{array}\right.
$$

Where, $\mathrm{S}_{\mathrm{t}}^{(3)}$ is the three exponential smoothing value for the time of $t ; S_{t-1}^{(3)}$ is the three exponential smoothing value for the time of $t-1$.

The three exponential smoothing is similar to the quadratic exponential smoothing, the displacement monitoring data is predicted by using the smoothing value to establish the forecasting model, and forecasting model is,

$$
Y_{t+T}^{(3)}=a_{t}+b_{t} T+c_{t} T^{2}
$$

Where, $Y_{t+T}^{(3)}$ is the three exponential smoothing predicting value of the forecast cycle $T$ for the time of $t$; $a_{t}, b_{t}$ and $c_{t}$ are smoothing coefficient, the formulas are,

$$
\left\{\begin{aligned}
a_{\mathrm{t}}= & 3 \mathrm{~S}_{\mathrm{t}}^{(1)}-3 \mathrm{~S}_{\mathrm{t}}^{(2)}+\mathrm{S}_{\mathrm{t}}^{(3)} \\
\mathrm{b}_{\mathrm{t}}= & \frac{\alpha}{2(1-\alpha)^{2}}\left[(6-5 \alpha) \mathrm{S}_{\mathrm{t}}^{(1)}-\right. \\
& \left.2(5-4 \alpha) \mathrm{S}_{\mathrm{t}}^{(2)}+(4-3 \alpha) \mathrm{S}_{\mathrm{t}}^{(3)}\right] \\
\mathrm{c}_{\mathrm{t}}= & \frac{\alpha^{2}}{(1-\alpha)^{2}}\left(\mathrm{~S}_{\mathrm{t}}^{(1)}-2 \mathrm{~S}_{\mathrm{t}}^{(2)}+\mathrm{S}_{\mathrm{t}}^{(3)}\right)
\end{aligned}\right.
$$

For the determination of value $\alpha$, its method is in accordance with the quadratic exponential smoothing. For the determination of the initial value, the following methods are used to select,

$$
\mathrm{S}_{0}^{(3)}=\mathrm{S}_{0}^{(2)}=\mathrm{S}_{0}^{(1)}=\mathrm{x}_{1}
$$

\section{ChaOs NeURAL NETWORK MODEL}

Based on the smoothing filter of the displacement data, chaos neural network forecasting method reprocess the displacement data by means of the concept of phase space reconstruction, use the improved $\mathrm{BP}$ neural network method to train the data, intellectually learn the changes laws, and finally, predictive analyze the displacement data.
Chaos neural network based on local-region method refers to the idea looking for adjacent points. Given a one dimensional nonlinear time sequence $Y_{M}(t)$, the number of input layer nodes can be determined by reconstructing phase space. And then training samples can be trained in neural network. Finally, the value of next point can be obtained by inputting prediction sample. This method is more complex and time-consuming than the former methods, while its calculation result is satisfying.

\section{A. Reconstract phase space}

The time-monitoring data curve is easy to obtain in geotechnical engineering, and it is a nonlinear time series. Since how these factors influence monitoring data is difficult to make clear, it is very important to build up a dynamic model to predict next value $x\left(t_{n+1}\right)$ according to one dimensional nonlinear time series: $x\left(t_{1}\right), x\left(t_{2}\right), L, x\left(t_{n}\right)$. There are many nonlinear time series prediction methods based on phase space reconstruction, which can be divide into whole-region method and local-region method according to attractor in fitting phase space.

For whole-region method, all the monitor data of the time series are use to predication the next value. For example, there is a monitor displacement time series $x_{1}, x_{2}, L, x_{n}$, let the $x_{1}, x_{2}, L, x_{n-1}\left(x_{i-1}\right)$ as independent variable and $x_{2}, x_{3}, L, x_{n}\left(x_{i}\right)$ as dependent variable, establish the relationship of $x_{i-1}$ and $x_{i}$, then can reconstruction of the monitor data, Fig. 3 shows an example of monitor data reconstruction result.

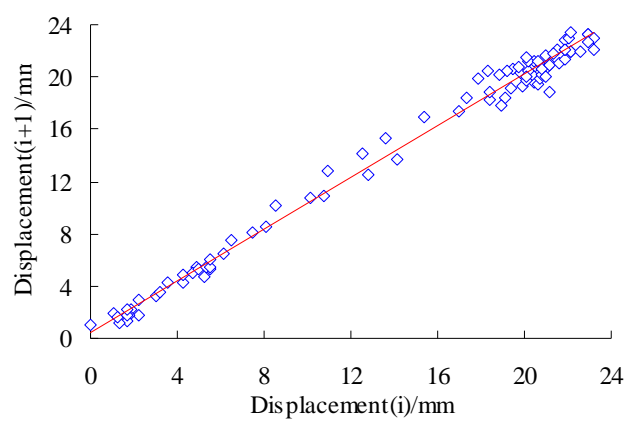

(a) Direction $\mathrm{x}$

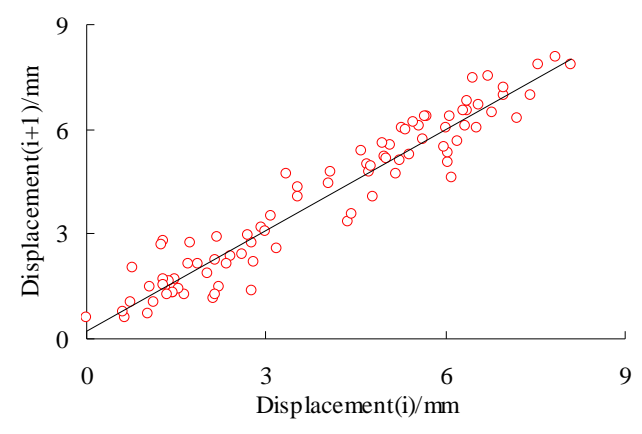

(b) Direction y

Figure 3. Reconstruction of the monitor data 
From Fig.3, the relationship of $x_{i-1}$ and $x_{i}$ is linear approximately, establish the fitting formula can used to predication next displacement value $x_{n+1}$.

In nonlinear dynamics, Packard et al have proposed to reconstruct phase space by using delay coordinate of a variable in initial system, and Takens have proposed to characterize the whole system by reconstructing phase space. Basic idea of phase space is: the Change of any variable in system is relative to other relative variables, the course of change of all these variables is the development process of whole system.

Here, define a unvaried time series $\left\{x\left(t_{i}\right), i=1, L, n\right\}, d$ is the dimension of attractor, then, $m \geq 2 d+1$. Delay time $\tau$ is determined by the mutual information method. Thus, the phase space of time series $\left\{x\left(t_{i}\right), i=1, L, n\right\}$ can be writed as follows,

$$
\begin{array}{r}
Y_{i}(t)=\left\{x\left(t_{i}\right), x\left(t_{i}+\tau\right), L, x\left(t_{i}+(m-1) \tau\right)\right\} \\
(i=1,2, L, M)
\end{array}
$$

\section{B. Seeking adjacent points}

In local-region method, the final point in phase space is regarded as a centre point and its adjacent points are regarded as correlative points, thus, the trajectory can be described according to correlative points and the next point can be predicted according to trajectory. Define nonlinear time series $Y_{M}(t)=\left\{Y_{1}, Y_{2}, L, Y_{M}\right\}$, final point $Y_{M}$ and adjacent points $j_{1}, j_{2}, L, j_{n}$, which are within a small neighborhood of final point $Y_{M}$, the fitting function can be obtained by employing least squares technique, and the next point can be predicted according to expression $Y_{M+1}=f\left(Y_{M}\right)$. Unlike whole-region method, local-region method predicts the next point according to final point and its adjacent points in nonlinear time series.

Let nonlinear time series $\mathrm{Y}_{\mathrm{M}}(\mathrm{t})=\left\{\mathrm{Y}_{1}, \mathrm{Y}_{2}, \mathrm{~L}, \mathrm{Y}_{\mathrm{M}}\right\}$, and $Y_{M}$ to be a centre point, then, adjacent points are defined as,

$$
\begin{aligned}
& \left|Y_{M}-Y_{j_{i}}\right| \leq\left|Y_{M}-Y_{M}-j_{i}\right| ; \\
& \quad\left(i=1,2, L, n ; n<M ; Y_{j_{i}} \in Y_{M}\right)
\end{aligned}
$$

Adjacent points can be selected according to the distance between centre point and adjacent points. For example, assuming that there are four adjacent points $Y_{1}, Y_{2}, Y_{3}, Y_{4}$, and only three adjacent points are needed, and the distance between centre point and adjacent points can be expressed as:

$$
\left|Y_{1}-Y_{M}\right|=\left|Y_{4}-Y_{M}\right| \geq\left|Y_{2}-Y_{M}\right| \geq\left|Y_{3}-Y_{M}\right|
$$

Then, adjacent points $Y_{2}, Y_{3}, Y_{4}$ will be chosen.

\section{Improved BP Neural Networks method}

Artificial Neural Networks are a powerful tool for prediction of nonlinearities. There are many kinds of ANN models, among which BPNN model is the most widely used [18]. The most common configuration of BPNN is composed of three layers. In a feed-forward network, the layers are completely connected to one another. As we know, the BPNN is easy run into the local-region minimum, in this paper, optimized the BPNN use Simulated Annealing algorithm [19, 20]. The optimized BPNN can jump off the local-region minimum, and get in the whole-region minimum.

Artificial neural network is a highly complex system that can simplify, abstract and simulate the human brain function; it has the capacity in memory, association, induction, summarize, abstract, self-organization, selflearning and self-adaptive. BP neural network is one of the most widely used artificial neural networks. Theory has proved: a three-layer BP network can approximate any mapping at arbitrary accuracy. A typical BP neural network structure has three layers: input layer, hidden layer and output layer.

$\mathrm{X}_{1}, \mathrm{X}_{2}, \mathrm{~L}, \mathrm{X}_{\mathrm{n}}$ are the input values of neural network, $C_{1}, C_{2}, L, C_{s}$ are the input values of the hidden layer, the output values are $y_{1}, y_{2}, L, y_{m}$, and the weighted value of the hidden layer neurons $j$ to the output neurons $k$ is $v_{j k}$. In addition, $o_{j}$ and $h_{k}$ are respectively represent bias values of the hidden layer and output layer.

Input of the units for the hidden layer is,

$$
c_{j}=f\left(\sum_{i=1}^{n} w_{i j} x_{i}+o_{j}\right) \quad(j=1,2, L, s)
$$

Output of the units for the output layer is,

$$
y_{k}=f\left(\sum_{j=1}^{s} v_{j k} c_{j}+h_{k}\right) \quad(k=1,2, L, m)
$$

And $f(z)$ is accord the Sigmiod function,

$$
f(z)=\frac{1}{1+\exp (-z)}
$$

In practical applications, BP neural network not only has slow convergence speed, but also its objective function has the local minimum. There are some good methods to improve the convergence speed, such as adding momentum, using anti-symmetric hyperbolic tangent function as activation function, but there are not good methods to solve the problem of local minimum. For example, in order to find the minimum objective function, the traditional BP algorithm uses the instantaneous gradient method to correct the weight vector. The amount of information is very small, it is prone to reach a local minimum, but it does not guarantee converging to the global minimum, so it require certain optimization algorithm to improve it.

In this paper, simulated annealing algorithm is used to improve the BP neural network, it is an extension of local search algorithm, and the basic idea is to find the global optimal solution of the optimization problem through simulating high-temperature objects annealing process. The cooling equation is used to cool the system,

$$
\mathrm{T}_{\mathrm{n}}=\mathrm{T}_{0} /(1+\ln \mathrm{n})
$$

Where, $T_{0}$ is the initial temperature; $n$ is the iteration step. 
In the cooling, the energy function is subject to Boltzmann Distribution,

$$
\mathrm{P}(\Delta \mathrm{E}) \propto \exp (-\Delta \mathrm{E} / \mathrm{T})
$$

Where, $P(\Delta E)$ is the probability of energy change; $T$ is the system temperature.

The simulated annealing process of the BP neural network is: weight vectors $w_{i j}$ and $v_{j k}$ is modified through a small perturbation of the current solution, then new weight vectors $\overline{w_{i j}}$ and $\overline{v_{j k}}$ are obtained, according to the current distribution of the error to determine whether or not to accept the current changes, energy function moves along the increasing direction when accepting the current changes, this process can separate the local minimum point from the solving process.

Based on simulated annealing algorithm, BP neural network uses it to speed up the convergence rate and avoid falling into the local minimum. The key of the learning process is to determine whether the neural network falling into a local minimum. Judgment is made by measuring the energy function whether or not to meet the accuracy requirement. If the value of energy function meets the accuracy requirement, the training is successful, if it does not, the neural network falls into a local minimum. Then simulated annealing algorithm can be used to jump out of the local minimum point.

\section{Predication model}

The processes of construction training samples and prediction in neural network will be presented in the following part of this paper.

(1) Constructing training samples

Given a one-dimensional nonlinear time sequence $Y_{M}(t)$, only the former $M-1$ data can be used to construct training samples. For a point $Y_{\mathrm{i}}$ (for $1<\mathrm{i} \leq \mathrm{M}-1$ ), look for adjacent points firstly, and then adjacent points can be input into neural network, and the next point $Y_{i+1}$ is the output of neural network.

(2) Neural network training

The training data are inputted into the neural network for training, the changes laws of the displacement data are learned intellectually through the neural network to obtain the changes laws of the displacement data of the slope, these can be used for the follow-up prediction analysis of the displacement.

(3) Forecast of neural network

To predict the value of point $Y_{M+1}$, an input sample can be constructed by seeking several adjacent points of point $Y_{M}$, and the value of point $Y_{M+1}$ is the output of neural network.

\section{CASE STUdY}

The above-mentioned exponential smoothing forecasting model and the chaos neural network model are used to predict the displacement monitoring data of the Three Gorges Ship-lock high slope.

\section{A. Engineering geology}

The two-way five-step Three Gorges Ship-lock, also known as the permanent Ship-lock, is an important part of the Three Gorges and is a navigation building with the world's highest navigation head and the largest scale. It is located on the left bank of the Yangtze River, 200m north of Tanzi ridge, the maximum distance from the Yangtze River waterfront is $1630 \mathrm{~m}$. The length of the Ship-lock line is $6442 \mathrm{~m}$, the length of the upstream channel is $2113 \mathrm{~m}$, lock chamber length is $1621 \mathrm{~m}$, and the downstream channel length is $2708 \mathrm{~m}$. There are 10 lock chambers and 12 lock gates, the total navigation head is $113 \mathrm{~m}$, million-ton fleet can pass it, annual capacity of one-way shipping is 50 million tons.

The scale of the two-way five-step Ship-lock is the largest in the world, its engineering characteristics are: high slope, complex structure, ground engineering and underground engineering are intertwined, and characteristics of high rock slope was particularly notable, so the slope stability is crucial. In order to ensure the stability of the slope during the excavation process, a variety of support measures are used to ensure the safety of the slope, at the same time the corresponding monitoring equipment is disposed at the key parts of the slope, including stress, seepage and degeneration monitoring.

The excavation of the Ship-lock high slope lasted 4 years, from November 1994 to the beginning of excavation to December 1999 to complete the basic excavation, excavation elevation is from $230 \mathrm{~m}$ to $96 \mathrm{~m}$, it is shown in Fig.4,

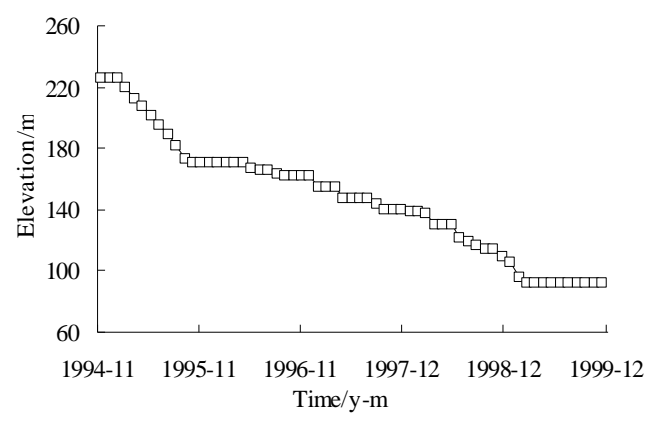

Figure 4. Excavation progress of ship-lock high slope

Fig. 5 shows the status of the Ship-lock high slope at different times, the shooting time is: the initial high-slope excavation, in April 1995; the basic end of excavation, in December 1998; and Ship-lock normal operation, in June 2003.

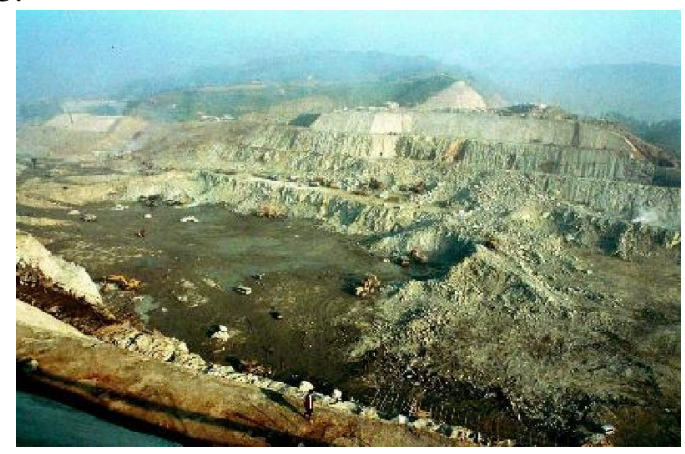


(a) Taken in April 1995

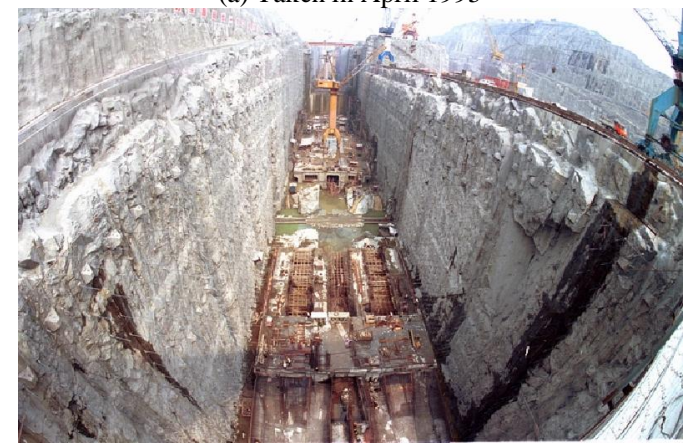

(b) Taken in December 1998

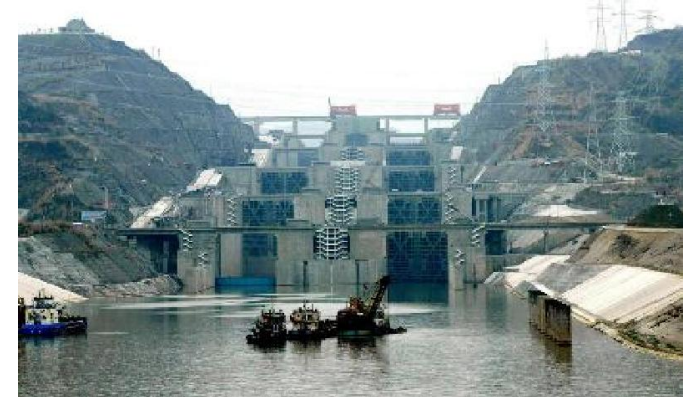

(c) Taken in June 2003

Figure 5. State of ship-lock high slope at different times

The chamber paragraph of the two-way five-step Shiplock is deeply excavated in the mountain, rock septal pier is reserved between gateway slot, its width is $54 \mathrm{~m} \sim 57 \mathrm{~m}$, the southern slope, the northern slope of the two high slope and the northern and southern slopes of the septal pier are formed after excavation, a total of four slopes. The elevation of the southern slope is $262.48 \mathrm{~m}$, the maximum height of $170 \mathrm{~m}$, the largest excavation depth of $174.50 \mathrm{~m}$ of the lock chamber. The excavation of the northern and southern slope is divided into two parts according to the gradient, the upper is the slope, gradient of the strong weathering zone is $1: 1$, gradient of the upper weak weathering zone is $1: 0.5$, and the bottom of the weak weathering zone and micro- new rock is $1: 0.3$, The berm is set up at intervals of $15 \mathrm{~m}$, the width is $5 \mathrm{~m}$, at the bottom of strong weathering zone set up a berm, the width is $15 \mathrm{~m} \sim 20 \mathrm{~m}$; The bottom is the vertical slope, constituting a Ship-lock chamber wall, at the top of the wall set up a platform, the width is $15 \mathrm{~m} \sim 30 \mathrm{~m}$. On both sides of the septal pier are also the vertical slopes.

\section{B. M onitor data of Slope displacement}

In order to carrying out safety monitoring for the slope stability, monitoring points are uniformly laid in accordance with cross-section layout. To reduce the instruments laid and observing workload, these crosssections are divided into key areas, important areas and general areas. Key monitoring parts have: the second chamber of the permanent Ship-lock and both sides of the high slope; the third chamber of the permanent Ship-lock and both sides of the high slope; the fifth lock gates of the permanent Ship-lock.

Many monitoring instruments are arranged for observation the deformation of high slope. Because of the hugeness of project work, complexity of construct techniques, prominence of synthetically benefit, there are several monitoring sections in this Ship-lock. In this paper, the displacement monitoring data of section 17-17 are forecast by employing the nonlinear time series predication methods. There are seven monitoring points to be arranged in South slope, three monitoring points in North Slope, and five monitoring points in the middle frusta.

Fig.6 is the plot plan. These above-mentioned methods are applied to predict the displacement of outer monitoring point TP/BM27 in the 17-17 section in high slope of Three Gorges Permanent Ship-lock.

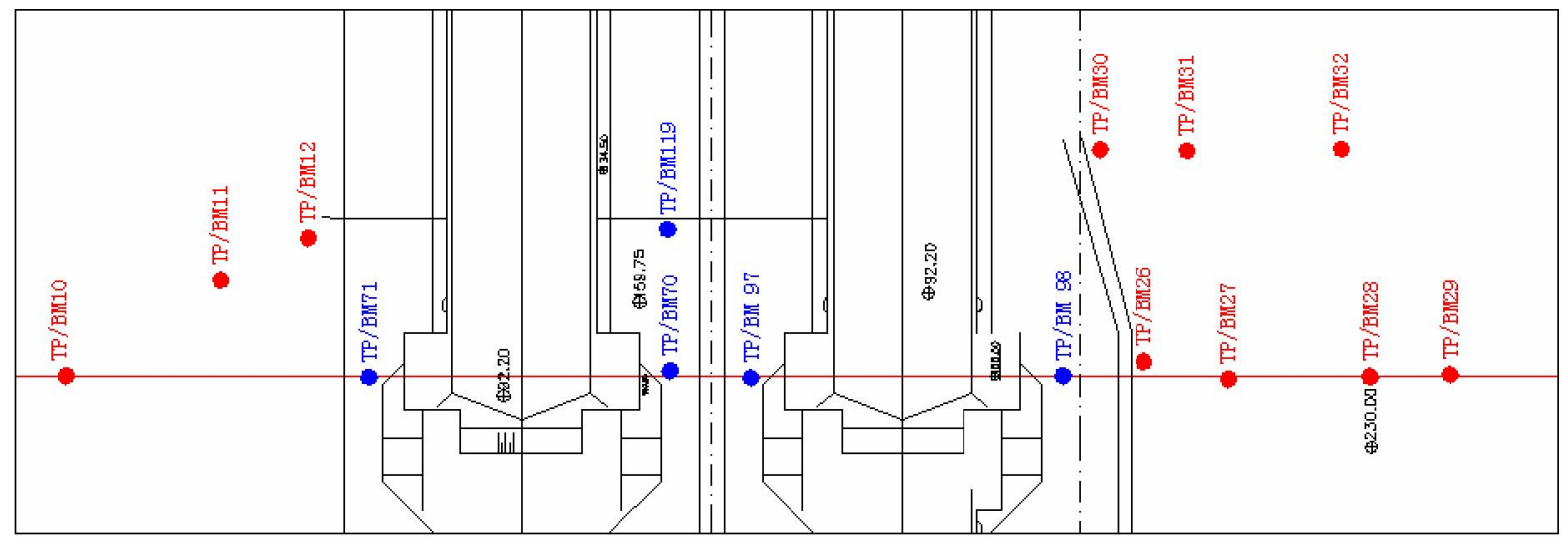

Figure 6. Disposal of the monitor point in section 17-17

The displacement of monitoring point TP/BM27 is plot in Fig.7. The total number of data points is 105 from November in 1995 to July in 2004, and the interval time is about one month. To guarantee the precision of prediction, the later 15 points are predicted and the former 90 points is viewed as known quantity. 


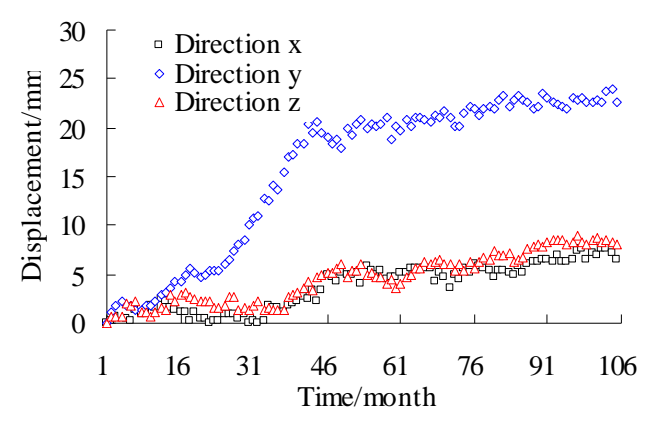

Figure 7. Nonlinear time series of monitor data in three directions

Where the $\mathrm{x}$ direction is along the river direction, the $\mathrm{y}$ direction is along the invading surface of the river, the $\mathrm{z}$ direction is the vertical direction. According to the displacement monitoring data of the three directions, the displacement of the $y$ direction is the largest, the displacement of the $\mathrm{x}$ and $\mathrm{z}$ directions is close in size. The changes laws of the slope displacement are mainly due to producing the excavation overhead surface, leading to the rock slope produced deformation to the overhead surface direction, And with the excavation going on, its displacement is increasing. As can be clearly seen from Fig.7, the growth rate of the displacement is fast during the construction period, after the completion of excavation, the increasing rate of the displacement in all directions is significantly reduced, degeneration of the slope become more and more stability.

\section{Displacement Data Preprocessing}

First, making credibility analysis for the displacement monitoring data, to determine their reliability and whether or not can be used to the predictive analysis process of the displacement. The displacement data credibility analysis results of the monitoring points in three directions are shown in Fig.8:

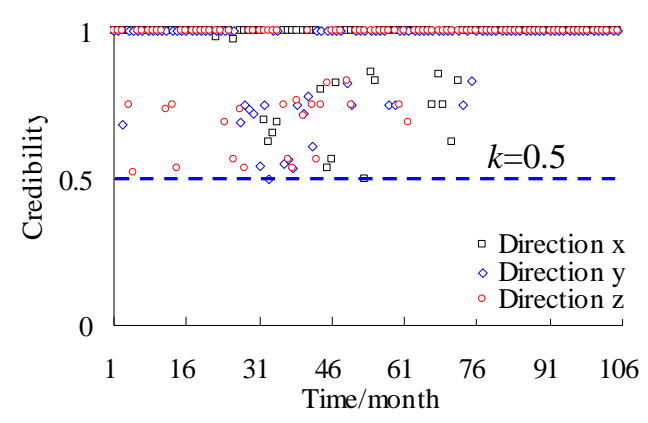

Figure 8. Credibility analysis of displacement data in three directions

As can be seen from Fig.8, the credibility of the displacement data in three directions are majority of 1.0, the credibility of all displacement data points are between $0.5 \sim 1.0$, in which the credibility reach 1.0 accounted for about $80 \%$, these explain that the displacement data of the monitoring points in three directions are reliable, it laid a good foundation for the follow-up forecast analysis.

Although the displacement data of the monitoring points in three directions have high credibility, some data points have large fluctuations and some errors, so smoothing filter method is used to process the displacement data, the results as shown in Fig.9:

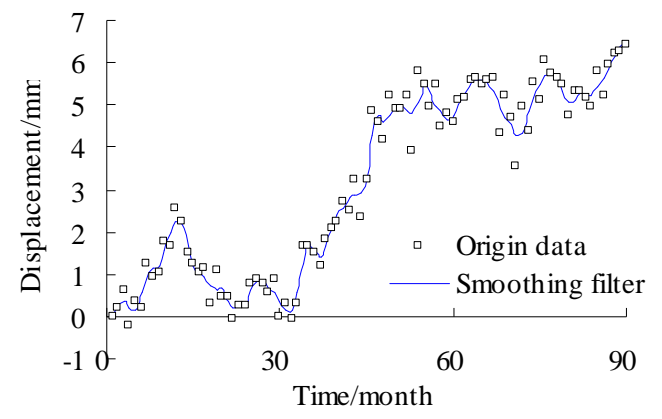

(a) Direction $\mathrm{x}$

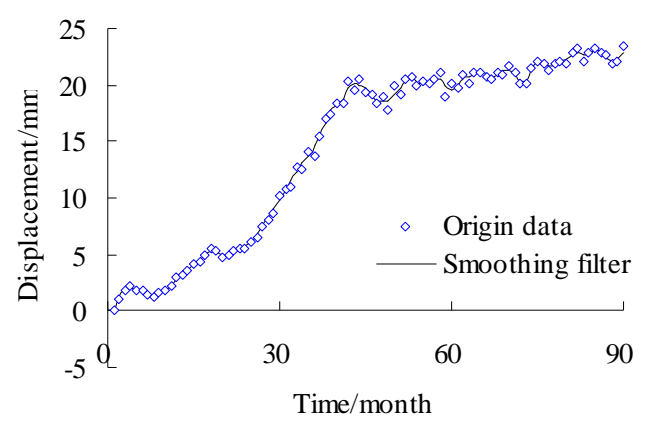

(b) Direction y

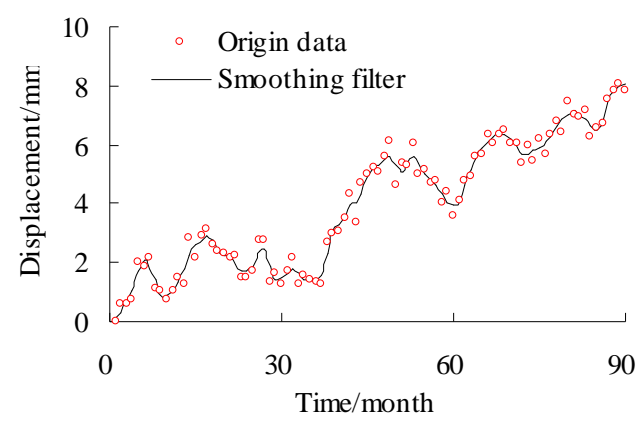

(c) Direction z

Figure 9. Smoothing filter of displacement data

As can be seen from Fig.9, for the displacement monitoring data in three directions, the displacement data in y direction is better, is can basically coincide with the original data after smoothing filter, and the displacement data in $\mathrm{x}$ direction is relatively poor, but after smoothing filter, some errors of the displacement monitoring data are removed, making monitoring data curve become more smooth, it make a great impact on the predict results, this influence will be concrete analyzed in the following chapter.

\section{Results of exponential smoothing method}

The quadratic exponential smoothing prediction method (QES method) and three exponential smoothing prediction method (TES method) are used to do predictive analysis of the displacement monitoring data in three directions, the displacement data after smooth filter can be used, the displacement of a total of 15 data points 
in $91 \sim 105$ months is analyzed, the displacement predictive results in all directions are shown in Fig.10.

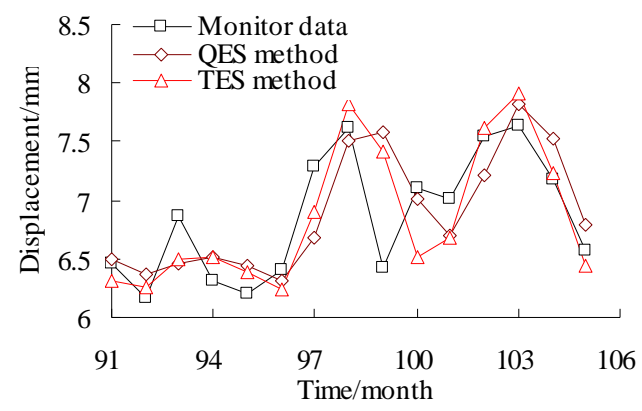

(a) Direction $x$

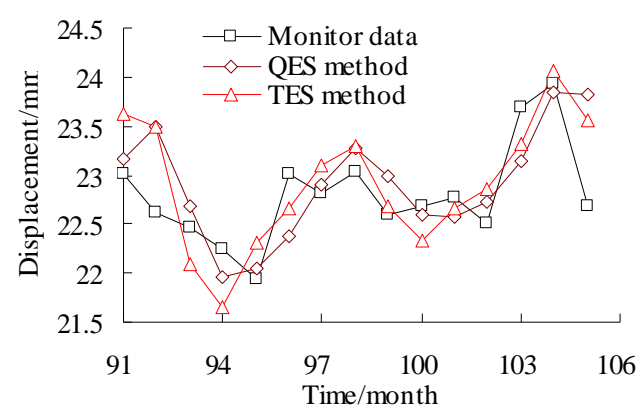

(b) Direction y

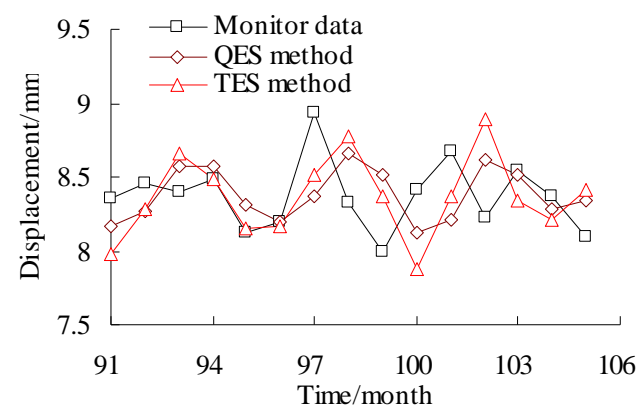

(c) Direction $\mathrm{Z}$
Figure 10. Predication results of exponential smoothing method

As can be seen from Fig.10, after smooth filter processing, the predictive results in all directions are close to the actual monitoring data, and the predictive results of the quadratic exponential smoothing method are close to three exponential smoothing method. This is mainly because the monitoring data basically coincide with the linear change; the quadratic exponential smoothing prediction method can meet the basic requirements. The results show that, exponential smoothing method apply to the predictive analysis of the displacement monitoring data of the slope.

\section{E. Results of C haos Neural Network method}

Using the chaos neural network method(CNN method) to predict the displacement, firstly, the training samples are build, the construction of the training samples also use the data that used smoothing filter processing, it included finding the neighboring points and then building the corresponding input and output, construction process of training samples is mentioned earlier.

Here only explaining displacement data prediction processing in the y direction. Training samples in Chaos Neural Network method are listed in Table I. There are three input vectors and an output vector in every training sample.

TABLE I.

TRAINING SAMPLES OF MONITORING POINT TP-BM27 IN Y DIRECTION

\begin{tabular}{|c|c|c|c|c|c|c|c|c|c|}
\hline Num. & \multicolumn{3}{|c|}{ Input } & \multirow{2}{*}{$\begin{array}{c}\text { Output } \\
1.73\end{array}$} & \multirow{2}{*}{$\begin{array}{c}\text { Num. } \\
46 \\
\end{array}$} & \multicolumn{3}{|c|}{ Input } & \multirow{2}{*}{$\begin{array}{c}\text { Output } \\
19.24\end{array}$} \\
\hline 1 & 1.87 & 2.21 & 1.07 & & & 19.51 & 20.41 & 19.42 & \\
\hline 2 & 1.74 & 1.87 & 2.21 & 1.34 & 47 & 19.07 & 19.42 & 19.51 & 20.43 \\
\hline 3 & 1.07 & 1.73 & 1.74 & 1.25 & 48 & 20.41 & 20.60 & 19.92 & 20.72 \\
\hline 4 & 1.34 & 1.07 & 1.73 & 1.68 & 49 & 19.92 & 19.51 & 19.42 & 20.37 \\
\hline 5 & 1.73 & 1.74 & 1.87 & 1.73 & 50 & 20.41 & 20.43 & 20.60 & 20.19 \\
\hline 6 & 1.73 & 1.74 & 1.68 & 2.20 & 51 & 20.37 & 20.41 & 20.43 & 20.47 \\
\hline 7 & 2.21 & 1.87 & 1.74 & 2.98 & 52 & 20.43 & 20.41 & 20.37 & 21.15 \\
\hline 8 & 2.21 & 2.20 & 1.87 & 3.17 & 53 & 20.72 & 20.60 & 20.47 & 18.87 \\
\hline 9 & 2.98 & 2.21 & 2.20 & 3.59 & 54 & 18.90 & 19.07 & 19.24 & 20.12 \\
\hline 10 & 3.17 & 2.98 & 2.21 & 4.23 & 55 & 20.19 & 19.92 & 20.37 & 19.74 \\
\hline$\ldots$ & $\ldots$ & $\ldots$ & $\ldots$ & $\ldots$ & $\ldots$ & $\ldots$ & $\ldots$ & $\ldots$ & $\ldots$ \\
\hline 36 & 17.35 & 16.99 & 15.36 & 18.29 & 81 & 22.92 & 22.89 & 23.22 & 22.61 \\
\hline 37 & 18.38 & 17.35 & 16.99 & 20.41 & 82 & 22.89 & 22.92 & 22.93 & 21.88 \\
\hline 38 & 18.38 & 18.29 & 17.35 & 19.51 & 83 & 21.87 & 21.89 & 21.86 & 22.12 \\
\hline 39 & 20.41 & 18.38 & 18.29 & 20.60 & 84 & 22.12 & 22.10 & 22.06 & 23.41 \\
\hline
\end{tabular}




\begin{tabular}{|l|l|l|l|l|l|l|l|l|l|}
\hline 40 & 20.41 & 19.51 & 18.38 & 19.42 & 85 & 23.24 & 23.22 & 22.93 & 23.02 \\
\hline 41 & 19.51 & 20.41 & 18.38 & 19.07 & 86 & 22.93 & 22.92 & 22.89 & 22.62 \\
\hline 42 & 19.42 & 19.51 & 18.38 & 18.38 & 87 & 22.61 & 22.89 & 22.92 & 22.47 \\
\hline 43 & 18.38 & 18.29 & 19.07 & 18.90 & 88 & 22.61 & 22.62 & 22.12 & 22.24 \\
\hline 44 & 19.07 & 18.38 & 18.38 & 17.84 & 89 & 22.12 & 22.12 & 22.10 & 21.93 \\
\hline 45 & 18.29 & 17.35 & 18.38 & 19.92 & 90 & 21.89 & 21.88 & 21.87 & 23.02 \\
\hline
\end{tabular}

The neural network is trained, the forecast data sample is inputted after training success, and then the corresponding results can be obtained. The predicted results of the chaos neural network in the y direction are shown in Fig. 11.

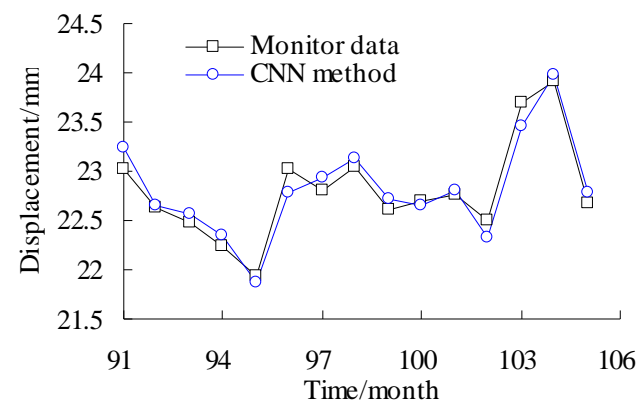

Figure 11. Predication result in direction y used CNN method

It is seen from Fig.11 that the prediction curve is basically consistent with the monitoring curve. It indicates Chaos Neural Network based on local-region method have an accurate prediction result.

\section{COMPARATIVE ANALYSIS OF PREDICATION METHODS}

Three forecasting methods are used to analyze the displacement monitoring data in high slope of the Three Gorges Ship-lock, the results are reasonable. In order to compare the performance of three forecasting methods, a comparative analysis of the results is carried out, and every prediction error is also analyzed. At the same time, the impact and role of the smoothing filter and the credibility of the site monitoring data on the results are analyzed.

\section{A. Comparison prediction results used different methods}

Three kinds of forecasting methods are used to predict and analyze the displacement monitoring data in the $y$ direction, the errors between the forecast results and the actual monitoring data are calculated, if the number of the forecast data is $m$, the formula for the error are as follows:

$$
\Delta \mathrm{y}_{\mathrm{i}}=\left|\mathrm{y}_{\mathrm{i}}-\mathrm{M}_{\mathrm{i}}\right| \quad(\mathrm{i}=1,2, \mathrm{~L}, \mathrm{~m})
$$

Where $\Delta y_{i}$ is the error of the point $i ; M_{i}$ is the displacement monitoring data; $y_{i}$ is the results of the models.

The prediction error of three kinds of methods is shown in Fig. 12.

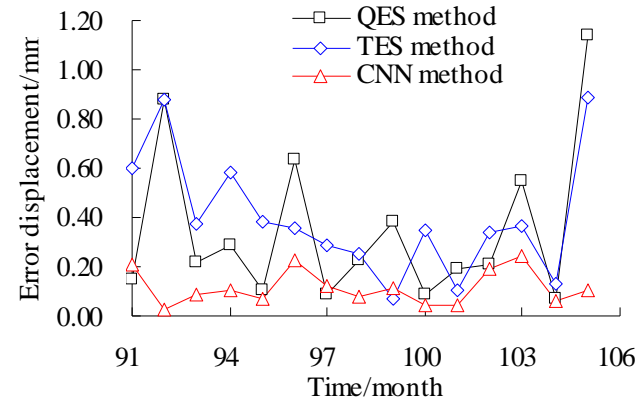

Figure 12. Predication error in direction y

As can be seen from Fig.12, the prediction error of the quadratic exponential smoothing method and three exponential smoothing methods are relatively large; the error of the chaos neural network method is very small. From the forecast results, the chaos neural network method is the best.

\section{B. Effect of monitor data filtering}

In order to analyzing the effects of data smoothing filter, the displacement results in the y direction are selected to do comparative analysis, it used the original monitoring data and smoothing filter data, the forecast results of different data are shown in Fig.13.

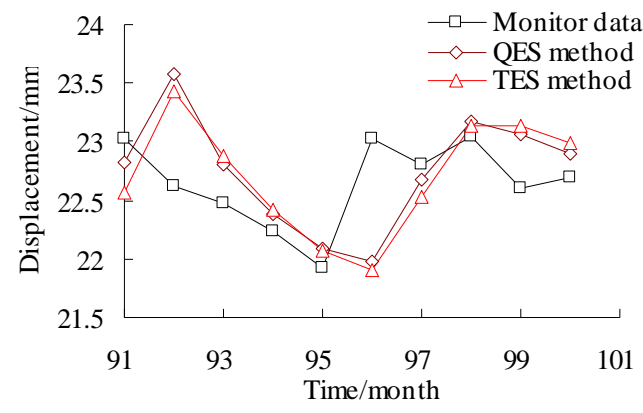

(a) Predication based on origin data

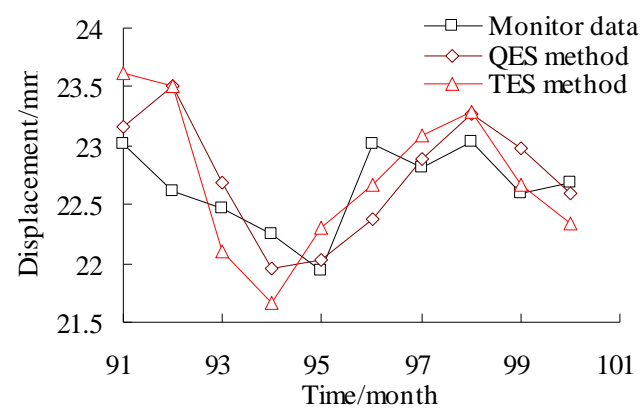

(b) Predication based on smoothing filter data

Figure 13. Predication results in direction y based on different data 
Fig.13(a) is the forecast results of the original monitoring data, Fig.13(b) is the forecast results of smoothing filter data. The forecast results with smoothing filter processing are significantly better than the results of the original monitoring data, the difference between the forecast results of the original monitoring data and the actual monitoring data is large, and forecast results with smoothing filter processing are basically consistent with the actual monitoring data. Through comparative analysis of the results, it shows that smoothing filter can effectively improve the accuracy of predictive results.

\section{Influnce of the data credibility}

In order to analyzing the impact of credibility of displacement monitoring data on the predictive results, ten data points of the monitoring data in three directions are predicted by using the quadratic exponential smoothing method and three exponential smoothing method, they are raw data that has not been used for smoothing filter.

The credibility analysis of displacement monitoring data shows that credibility in three directions is: the credibility in the $\mathrm{y}$ direction is the best; in the $\mathrm{z}$ direction comes second; and in the $\mathrm{x}$ direction is the worst. The credibility of the displacement data has certain impact on the rationality of the results; forecast results in three directions are shown in Fig.14,

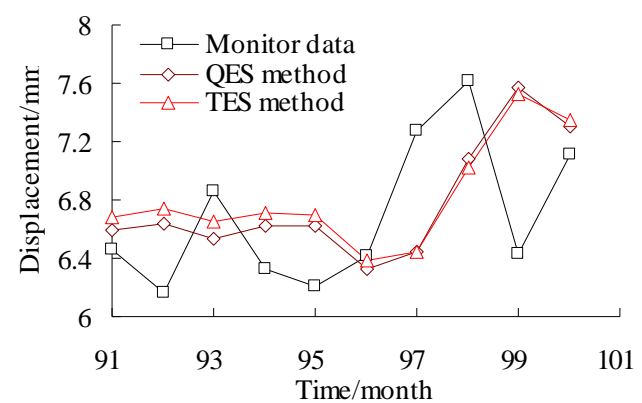

(a) Direction $x$

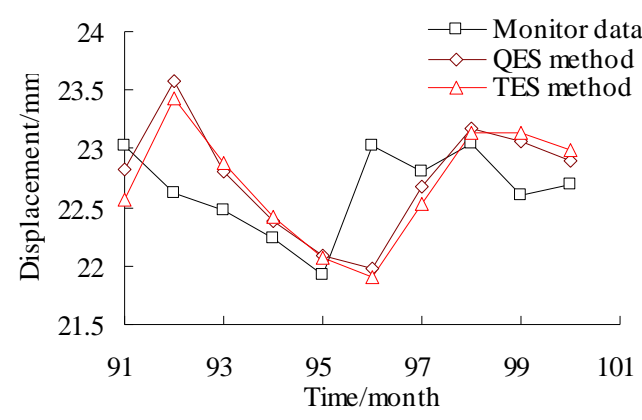

(b) Direction y

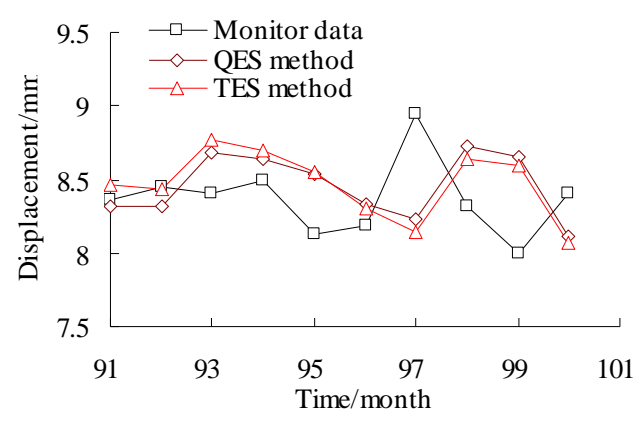

(c) Direction z

Figure 14. Predication results based on origin monitor data

As can be seen from Fig.14, in y direction the difference between the forecast results and the actual monitoring data is small, in $\mathrm{z}$ direction is better, and in $\mathrm{x}$ direction is the largest. The forecast results show that the better of the data credibility, the more desirable of the results, in contrast, using the poor reliable data will affect the reasonableness of the forecast and may lead to forecast failure.

\section{CONCLUSION}

Nonlinear time series analysis and forecasting is an active research area over the last few decades. The accuracy of nonlinear time series forecasting is fundamental to many decision processes and hence the research for improving the effectiveness of forecasting models has never stopped.

For the displacement monitoring data at the scene, the monitoring data will have errors because of a variety of reasons; it has great impact on the displacement forecast results. In order to eliminating these effects, it is necessary to carry out pre-processing for the displacement monitoring data. Before the pre-processing, the credibility can be used to analyze the reliability of monitoring data, so reliability of monitoring data is obtained. In this paper, smoothing filter method is used; its characteristic is simple and practical.

Based on the smoothing filter of the displacement data, three methods of the forecast analysis for the slope are provided: the quadratic exponential smoothing method, the three exponential smoothing methods and chaos neural network. And they are used to analyze the displacement monitoring data of the high slope of the Three Gorges Ship-lock, it obtained a better predictive results.

The predictive results of these three methods are comparative analyzed, and the result of the chaos neural network is the best, at the same time, the better of the data credibility, and the more desirable of the results. For the role of the smoothing filter, it can effectively reduce the error of the monitoring data and achieve better results.

\section{ACKNOWLEDGMENT}

This work was supported by Project 973 of Chinese National Program of Basic Research (No. 2002CB412707) 
and Chinese National Natural Science Foundation (No. 50539110).

\section{REFERENCES}

[1] D. Pena and J. Rodriguez, "Detecting nonlinearity in time series by model selection criteria," International J ournal of Forecasting, vol. 21, pp. 731-748, April 2005.

[2] G. P. Zhang, "Time series forecasting using a hybrid ARIMA and neural network model," Neurocomputing, vol. 50, pp. 159-175, January 2003.

[3] F. Takens, Detecting strange attractors in fluid turbulence. In: Rand, D. A., \& Young, L. S. (Eds.), D ynamical Systems and Turbulence, Lecture Notes in Mathematics. Berlin: Springer-Verlag, 1980.

[4] A Wolf, J. B. Swift, H. L. Swinney, et al, "Determining Lyapunov exponents from a time series," Physica D, vol. 16, pp. 285-317, March, 1985.

[5] R. Hodrick, E. Prescott, Post-war US business cycles: an empirical investigation, Working paper, Carnegie-Mellon University, 1980.

[6] M. Baxter, R. King, Measuring business cycles: approximate band-pass filters for economic time series, NBER working paper 5022, 1995.

[7] L. D. Yang, Theory of back analysis and engineering application of geotechnical engineering problem. Beijing: Science Press, 1996.

[8] M. E. Torresa and L. G. Gamero, "Relative complexity changes in time series using information measures," Physica A, vol 286, pp. 457-473, November 2000.

[9] G. P. Zhang, B. E. Patuwo and M.Y. Hu, "A simulation study of artificial neural networks for nonlinear time-series forecasting," Computers \& O perations Research, vol. 28, pp. 381-396, April 2001.

[10] J. Timmer, "Parameter estimation in nonlinear stochastic differential equations," Chaos, Solitons and Fractals, vol. 11, pp. 2571-2578, December 2000.

[11] R. M. Ramiro, A. A. Raymond and G. K. Ioannis, "Noninvertibility in neural networks," Computers and Chemical Engineering, vol. 24, pp. 2417-2433, Novembe 2000.

[12] J. Timmer, H. Rust, W. Horbel, et all, "Parametric, nonparametric and parametric modelling of a chaos circuit time series," Physics Letters A, vol. 274, pp. 123-134, September 2000.

[13] R. S. Norman and W. Halbert, "Forecasting economic time series using flexible versus fixed specification and linear versus nonlinear economic models," International J ournal of Forecasting, vol. 13, pp. 439-461, December 1997.

[14] C. W. Chu and G. P. Zhang, "A comparative study of linear and nonlinear models for aggregate retail sales forecasting," International Journal of production economics, vol. 86, pp. 217-231, December 2003.
[15] Z. Landsman, U. E. Makov, "Credibility evaluation for the exponential dispersion family," Insurance: Mathamatics and Economics, vol. 24, pp. 23-29, January 1999.

[16] S. L. Sun, "Multi-sensor information fusion white noise filter weighted by scalars based on Kalman predictor," Automatica, vol. 40, pp. 1447-1453, September 2004.

[17] X. Hu, D. V. Prokhorov, D. C. Wunsch, "Time series prediction with a weighted bidirectional multi-stream extended Kalman filter," Neurocomputing, vol. 70, pp. 2392-2399, November 2007.

[18] H. B. Wang, W. Y. Xu and R. C. Xu., "Slope stability evaluation using Back Propagation Neural Networks," Engineering G eology, vol. 80, pp. 302-315, August 2005.

[19] J. W. Zhou, W. Y. Xu, A. C. Shi, "Application of nonlinear time series analysis to excavation deformation predication of high slope," Chinese Journal of Rock Mechanics and Engineering, vol. 25, pp. 2795-2800, June 2006.

[20] S. S. Randall, E. D. Robert and D. J. John, "Optimization of neural networks: A comparative analysis of genetic algorithm and simulated annealing," European Journal of Operational Research, vol. 114, pp. 589-601, May 1999.

J iawen Zhou was born in Ji-an, Jiangxi, China in 1982. He received the B.S. degree in architecture engineering form East China Jiaotong University in 2003; and the M.S. and Ph.D. degree in geotechnical engineering from Hohai University in 2005 and 2008, respectively. His research interests in Geotechanical mechanics and engineering. Dr. Jiawen Zhou is an IAEG Member, a member of China Society of Rock Mechanics and Engineering, Chinese Society of Theoretical and Application Mechanics, China Civil Engineering Society, Chinese Society of Hydropower Station, Chinese Society of Hydro-electric power.

Xingguo $\mathrm{Y}$ ang received the B.S. degree in hydropower project construction form the Chengdu University of Science and Technology in 1991; and the M.S. and Ph.D. degree in hydraulic structural engineering from Sichuan University in 1998 and 2008, respectively. His research interests in hydraulic structural engineering. Dr. Xingguo Yang is an IAEG Member, a member of Chinese Society of Hydropower Station, Chinese Society of Hydro-electric power.

Wei $\mathrm{Hu}$ received the B.S. degree in hydraulic and hydropower engineering from Sichuan University, in 2008. She is M.S. student at Sichuan University and majors in hydraulic structure engineering. Her research interests in involves stability analysis, construction technology research and numerical calculation of the underground engineering under complicated geological conditions. 